\title{
SEGMENTATION OF POLARIMETRIC SAR DATA BASED ON THE FISHER DISTRIBUTION FOR TEXTURE MODELING
}

Lionel Bombrun

\author{
Grenoble Image Parole Signal et Automatique \\ CNRS INPG - 961, Rue de la Houille Blanche \\ BP 46 - 38402 Saint-Martin-d'Hères, France \\ lionel.bombrun@gipsa-lab.inpg.fr
}

\author{
Jean-Marie Beaulieu
}

\author{
Laval University
}

Computer Science and Software Engineering Department Québec City, QC, G1K-7P4, Canada

jean-marie.beaulieu@ift.ulaval.ca

\begin{abstract}
The Polarimetric Synthetic Aperture Radar (PolSAR) covariance matrix is generally modeled by a complex Wishart distribution. For textured scenes, the product model is used and the texture component is often modeled by a Gamma distribution. In this paper, authors propose to use the Fisher distribution for texture modeling. From a Fisher distributed texture component, we derive the distribution of the complex covariance matrix and we propose to implement the KummerU distribution in a hierarchical segmentation and a hierarchical clustering algorithm. Segmentation and classification results are shown on synthetic images and on ESAR L-band PolSAR data over the Oberpfaffenhofen test-site.
\end{abstract}

Index Terms - Polarimetric SAR images, Fisher distribution, Texture, KummerU, Classification, Segmentation

\section{INTRODUCTION}

SAR data are the result of a coherent imaging system that produces the speckle noise phenomenon. If the antenna can emit and receive vertically and horizontally polarized waves, the covariance matrix is used to characterize the interactions between the electromagnetic wave and the scatterers. For fully developed speckle, the covariance matrix follows the complex Wishart distribution [1]. It is assumed that land cover backscatter characteristics are homogeneous (uniform or not textured). For textured scenes, the "scalar product model" has been proposed [2]. The observed signal is the product of a positive scalar texture component $\mu$ (polarization independent) with the speckle component. The texture term is generally modeled by a Gamma distribution and the covariance matrix follows a $\mathcal{K}$ distribution.

In this paper, we propose to use a more general probability density function (pdf) for the texture parameter: the Fisher distribution. Then, with the scalar product model, we show that the pdf of the observed covariance matrix can be expressed in term of the KummerU confluent hypergeometric function of the second kind. Then, we propose to implement this new pdf in a hierarchical segmentation algorithm. Segmentation results are illustrated on a synthetic image. Finally, the KummerU pdf is implemented in a totally unsupervised hierarchical classification algorithm. Classification results are shown on ESAR L-band PolSAR data over the Oberpfaffenhofen test-site.

\section{COVARIANCE MATRIX DISTRIBUTION}

\subsection{Pdf for homogeneous scenes}

One-look PolSAR data are completely characterized by the scattering matrix $\mathbf{S}=\left[\begin{array}{ll}S_{h h} & S_{h v} \\ S_{v h} & S_{v v}\end{array}\right] . \quad S_{h v}$ is the scattering component for horizontally polarized transmitting antenna and vertically polarized receiving antenna. For the monostatic case, the reciprocity assumption holds. The cross-polarization terms $S_{h v}$ and $S_{v h}$ are equal. The radar backscattering signal is described by the target scattering vector $\mathbf{x}=\left(S_{h h}, S_{v v}, \sqrt{2} S_{h v}\right)^{T}$ where $\mathrm{T}$ is the transposition operator. For homogeneous (no textured) scene (denoted by subscript $\mathrm{h}$ ) and fully developed speckle, the pdf of $\mathbf{x}$ follows a zero mean multivariate complex Gaussian distribution [1]. The L-look covariance matrix, $\mathbf{Z}_{h}=(1 / L) \sum_{k=1}^{L} \mathbf{x}_{k} \mathbf{x}_{k}^{H}$, follows the complex Wishart distribution [1]

$$
p_{\mathbf{Z}_{h}}\left(\mathbf{Z}_{h} \mid \Sigma_{h}\right)=\frac{L^{L p}\left|\mathbf{Z}_{h}\right|^{L-p} \exp \left\{-L \operatorname{tr}\left(\Sigma_{h}{ }^{-1} \mathbf{Z}_{h}\right)\right\}}{\pi^{\frac{p(p-1)}{2}} \Gamma(L) \cdots \Gamma(L-p+1)\left|\Sigma_{h}\right|^{L}}
$$

where $\Sigma_{h}=E\left[\mathbf{x x}^{H}\right]$ is the population covariance matrix. $E[\cdot], \operatorname{tr}(\cdot)$ and $|\cdot|$ are respectively the expectation, the trace and the determinant operator. The superscript $H$ denotes the complex conjugate transposition. $p$ is the dimension of the target scattering vector $\mathbf{x}$ ( $p=3$ for the reciprocal case).

\subsection{The product model}

For textured areas, the observed covariance matrix $\mathbf{Z}$ can be written as the product of a texture parameter $\mu$ with the covariance matrix for homogeneous surface, $\mathbf{Z}=\mu \mathbf{Z}_{h}$. Generally 
the texture is polarimetric dependent and $\mu$ is represented by a matrix. Here, $\mu$ is assumed to be a positive scalar parameter. The probability density function of the covariance matrix $\mathbf{Z}$ can be derived by the following equation [2] [3] [4]:

$$
p_{\mathbf{Z}}\left(\mathbf{Z} \mid \Sigma_{h}, \alpha\right)=\int_{0}^{\infty} p_{\mathbf{Z}_{h}}\left(\mathbf{Z} \mid \mu \Sigma_{h}\right) p_{\mu}(\mu \mid \alpha) d \mu
$$

where $\alpha$ is the parameter set of texture distribution.

For forest areas, the Gamma distribution has been widely used to model the texture. The associated covariance matrix follows a $\mathcal{K}$ distribution, so named because it uses the modified K-Bessel function of the second kind [3].

With high resolution images of man-made objects, the Fisher distribution has been successfully introduced to model the SAR clutter [5]. In next section, the advantage of this distribution family for texture modeling is examined and the covariance matrix pdf is derived analytically.

\section{SCENE TEXTURE DISTRIBUTION}

\subsection{Fisher distribution}

The pdf of the Fisher distribution is defined by 3 parameters as:

$$
p_{\mu}(\mu)=\frac{\Gamma(\mathcal{L}+\mathcal{M})}{\Gamma(\mathcal{L}) \Gamma(\mathcal{M})} \frac{\mathcal{L}}{\mathcal{M} m} \frac{\left(\frac{\mathcal{L} \mu}{\mathcal{M} m}\right)^{\mathcal{L}-1}}{\left(1+\frac{\mathcal{L} \mu}{\mathcal{M} m}\right)^{\mathcal{L}+\mathcal{M}}}
$$

with $\mathcal{L}>0, \mathcal{M}>0$. The Fisher distribution is not related to electromagnetic wave theory. It involves the Mellin transform and second kind statistics.

\subsection{Benefit of Fisher distribution}

Fig. 1 shows Gamma, Inverse Gamma and Fisher pdf in the $\kappa_{2} / \kappa_{3}$ plan introduced by [6]. Gamma and Inverse Gamma pdf are respectively represented by the blue and red line. Fisher pdf cover all the space between the blue and red line. As Fisher distribution is equal to the Mellin convolution of a Gamma distribution by an inverse Gamma distribution, it can be viewed as a generalization of the Gamma distribution.

\subsection{KummerU distributed covariance matrix}

As the Fisher distribution fit well a large range of SAR clutter [5], it is of great interest to derive the covariance matrix pdf for a Fisher distributed texture. This pdf is shown in Eq. 4, it is obtained by substituting Eq. 1 and Eq. 3 into Eq. 2. All the mathematical details can be found in [7].

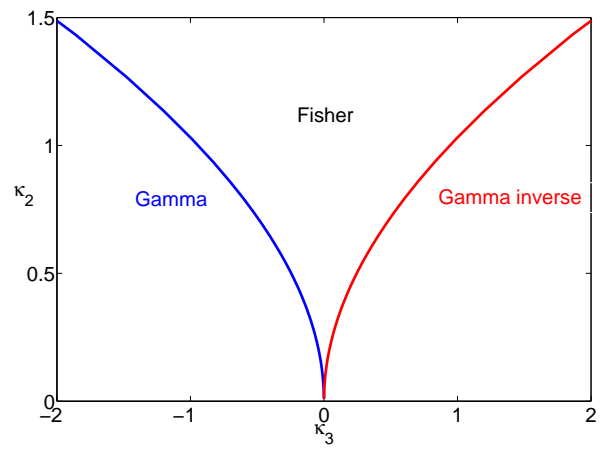

Fig. 1. Gamma, Inverse Gamma and Fisher pdf in the $\kappa_{2} / \kappa_{3}$ plan

$$
\begin{aligned}
& p_{\mathbf{Z}}\left(\mathbf{Z} \mid \Sigma_{h},\right.\mathcal{L}, \mathcal{M}, m)=\frac{L^{L p}|\mathbf{Z}|^{L-p}}{\pi^{\frac{p(p-1)}{2}} \Gamma(L) \cdots \Gamma(L-p+1)\left|\Sigma_{h}\right|^{L}} \\
& \times \frac{\Gamma(\mathcal{L}+\mathcal{M})}{\Gamma(\mathcal{L}) \Gamma(\mathcal{M})}\left(\frac{\mathcal{L}}{\mathcal{M} m}\right)^{L p} \Gamma(L p+\mathcal{M}) U(a, b, z)
\end{aligned}
$$

The covariance matrix distribution of a Fisher distributed clutter can be expressed as a function of the confluent hypergeometric function of the second kind KummerU (denoted $U(\cdot, \cdot, \cdot))$ with $z=\frac{L \operatorname{tr}\left(\Sigma_{h}{ }^{-1} \mathbf{Z}\right) \mathcal{L}}{\mathcal{M} m}, a=L p+\mathcal{M}$ and $b=1+L p-\mathcal{L}$. In the following, such pdf will be named KummerU distribution.

\section{HIERARCHICAL SEGMENTATION}

In this paper, the hierarchical segmentation algorithm proposed by Beaulieu and Touzi [8] is adapted to the KummerU distributed covariance matrix. The segmentation process can be divided into three steps:

1. Definition of an initial partition.

2. For each 4-connex segments pair, the stepwise criterion, SC, is computed. Then, we find and merge the two segments which minimize the criterion.

3. Stop if the maximum number of merges is reached, otherwise go to step 2.

\subsection{Stepwise criterion}

The criterion used in the hierarchical algorithm is based on the log-likelihood function. The hierarchical algorithm merges the two adjacent segments $S_{i}$ and $S_{j}$ which minimizes the loss of likelihood. The stepwise criterion $\left(\mathrm{SC}_{i, j}\right)$ can be expressed as [8]:

$$
\operatorname{SC}_{i, j}=\operatorname{MLL}\left(S_{i}\right)+\operatorname{MLL}\left(S_{j}\right)-\operatorname{MLL}\left(S_{i} \cup S_{j}\right)
$$




\begin{tabular}{|c|c|}
\hline texture 1 & texture 2 \\
$\mathcal{L}=5$ & $\mathcal{L}=5$ \\
$\mathcal{M}=10$ & $\mathcal{M}=30$ \\
$m=1$ & $m=1$ \\
& \\
\hline texture 3 & texture 4 \\
$\mathcal{L}=10$ & $\mathcal{L}=10$ \\
$\mathcal{M}=10$ & $\mathcal{M}=30$ \\
$m=1$ & $m=1$ \\
& \\
\hline
\end{tabular}

(a)

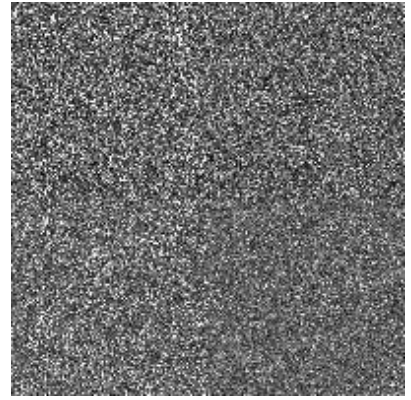

(b)
Fig. 2. (a) image containing the 4 segments (ground truth) and Fisher parameters used in the simulation (b) 4-areas synthetic texture image $(200 \times 200)$

where $\operatorname{MLL}(\cdot)$ is the segment maximum log-likelihood function.

For the KummerU distribution, the maximum log-likelihood function for segment $S$ is derived from Eq. 4. After removing terms that will be cancelled in the stepwise criterion, the log-likelihood function can be rewritten as

$$
\begin{aligned}
& \operatorname{MLL}(S)=-n L \ln \left|C_{h}\right|+n \ln \{\Gamma(\hat{\mathcal{L}}+\hat{\mathcal{M}})\} \\
& -n \ln \{\Gamma(\hat{\mathcal{L}})\}-n \ln \{\Gamma(\hat{\mathcal{M}})\}+n L p \ln \left(\frac{\hat{\mathcal{L}}}{\hat{\mathcal{M}} \hat{m}}\right) \\
& +n \ln \{\Gamma(L p+\hat{\mathcal{M}})\} \\
& +\sum_{\mathbf{Z}_{k} \in S} \ln \left\{\mathrm{U}\left(L p+\hat{\mathcal{M}} ; 1+L p-\hat{\mathcal{L}} ; \frac{L \operatorname{tr}\left(C_{h}^{-1} \mathbf{Z}_{k}\right) \hat{\mathcal{L}}}{\hat{\mathcal{M}} \hat{m}}\right)\right.
\end{aligned}
$$

\subsection{Segmentation result}

The evaluation of segmentation result is a difficult problem. Fig.3 presents curves similar to Receiver Operator Character$\int_{p_{d}}$ versus the false alarm probability $p_{f a}$. At each iteration, (69 new partition is produced by merging 2 segments. ROC where each segment is a bloc of $10 \times 10$ pixels. The initial partition is composed of 400 segments. istic (ROC) curves. ROC curves plot the detection probability curves are obtained by calculating $p_{d}$ and $p_{f a}$ for each partition. A description of the method to calculate $p_{d}$ and $p_{f a}$ from a partition $P$ can be found in [7].

In Fig. 3, the ROC curve for the KummerU distribution is better than the curves for the Wishart distribution and $\mathcal{K}$ distribution. When $p_{f a}=0.05$, the detection probability is much better for the KummerU criterion (0.85) than for the Wishart and $\mathcal{K}$ distribution criterion (0.3) and the corresponding partitions, shown in Fig.4, contain respectively 5, 29 and 11 segments. This shows the advantage of using a criterion that can discriminate between a large range of texture distributions.

\section{HIERARCHICAL CLASSIFICATION}

From an over-segmented partition $P$ of $K$ segments given by the hierarchical segmentation algorithm, a classified image can be obtained by using a hierarchical clustering algorithm. First of all, only the $L$ largest segments (named clusters) of the over-segmented partition are selected. Then, at each iteration, the two more similar clusters are merged. The criterion

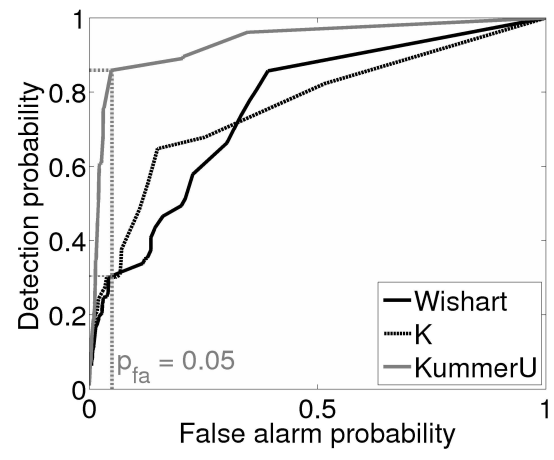

Tab. 1 shows the Kolmogorov distance between the Fisher istributions used in the simulation. Note that this distance simulated data is a difficult task because the same Wishart distribution is used and texture are quite similar, as can be observed in Fig. 2(b)

Table 1. Kolmogorov distance between the 4 textures.

\begin{tabular}{|c|c|c|c|c|c|c|}
\hline \hline textures & $1-2$ & $1-3$ & $1-4$ & $2-3$ & $2-4$ & $3-4$ \\
\hline$d_{K}$ & 0.049 & 0.074 & 0.102 & 0.063 & 0.092 & 0.072 \\
\hline \hline
\end{tabular}




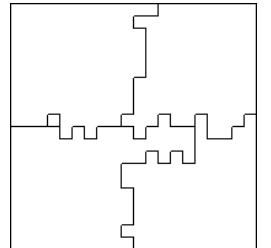

(a)

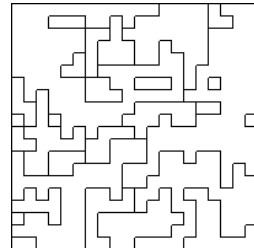

(b)

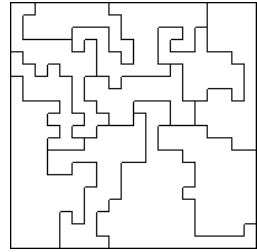

(c)
Fig. 4. Segmentation for a given false alarm probability $p_{f a}=0.05$ for (a) KummerU criterion ( 5 segments), (b) Wishart criterion (29 segments), (c) $\mathcal{K}$ criterion (11 segments)

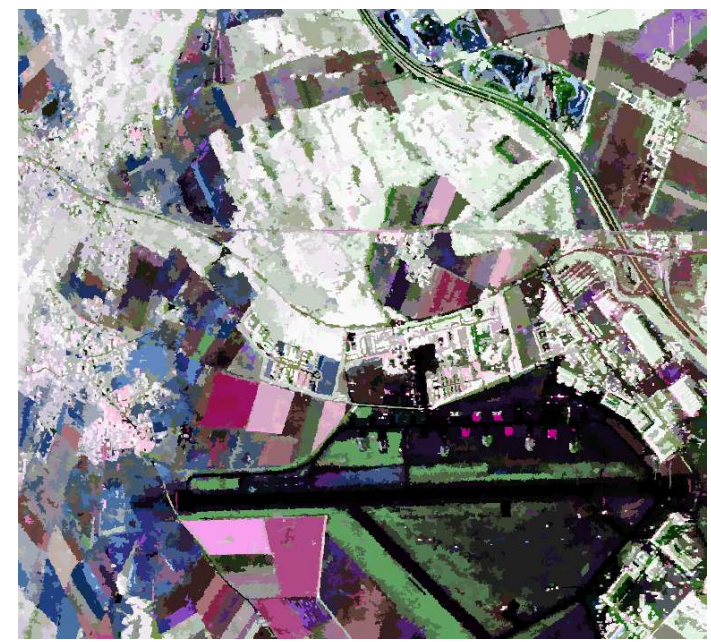

Fig. 5. 100 classes

used by the algorithm is based on the KummerU distribution. Here, there is no neighborhood restraint of 4-connexity. Clusters are merged iteratively until to reach the desired number of classes. Finally, the $(K-L)$ small segments are merged to the nearest class for the Wishart distance sense.

The proposed unsupervised classification algorithm is applied on the well known 8-look L-band PolSAR image over the Oberpfaffenhofen test site. First, the hierarchical segmentation algorithm is applied to segment the image into 10000 segments. Then, the 3567 largest clusters ( $\geq 20$ pixels) are merged iteratively. Fig. 5 shows a colored composition in the Pauli basis of the classification obtained on the Oberpfaffenhofen image for 100 classes. High resolution details are well preserved in the classification map. For example, in urban areas, streets and buildings are isolated.

\section{CONCLUSION}

In this paper, the Fisher distribution has been used to model the texture of PolSAR data. The Fisher pdf provide a better texture modeling than the Gamma pdf. With the product model hypothesis, the covariance matrix distribution for a Fisher textured clutter has been mathematically established and expressed as a function of the confluent hypergeometric function of the second kind (KummerU). Next, this distribution has been implemented in a hierarchical segmentation algorithm by calculating the log-likelihood function. Comparisons with the Wishart and $\mathcal{K}$ distributions have been done for synthetic textured scenes. ROC curves reveal that the use of an appropriate texture distribution is useful to segment textured PolSAR images.

Finally, an unsupervised hierarchical classification algorithm has been proposed and applied on ESAR L-band PolSAR data.

\section{REFERENCES}

[1] N.R. Goodman, "Statistical analysis based on a certain multivariate complex Gaussian distribution (an introduction)," in Ann. Math. Statist., 1963, vol. 34, pp. 152-177.

[2] I.R. Joughin, D.P. Winebrenner, and D.B. Percival, "Polarimetric Density Functions for Multilook Polarimetric Signatures," IEEE Transactions on Geoscience and Remote Sensing, vol. 32, no. 3, pp. 562-574, 1994.

[3] J.S. Lee, D.L. Schuler, R.H. Lang, and K.J. Ranson, "K-Distribution for Multi-Look Processed Polarimetric SAR Imagery," in Geoscience and Remote Sensing, IGARSS '94, Pasadena, California, United States, 1994, pp. 2179-2181.

[4] A. Lopès and F. Séry, "Optimal Speckle Reduction for the Product Model in Multilook Polarimetric SAR Imagery and the Wishart Distribution," IEEE Transactions on Geoscience and Remote Sensing, vol. 35, no. 3, pp. 632-647, 1997.

[5] C. Tison, J.-M. Nicolas, F. Tupin, and H. Maître, "A New Statistical Model for Markovian Classification of Urban Areas in High-Resolution SAR Images," IEEE Transactions on Geoscience and Remote Sensing, vol. 42, no. 10, pp. 2046-2057, October 2004.

[6] J.-M. Nicolas, "Introduction aux Statistiques de deuxième espèce: applications des Logs-moments et des Logs-cumulants à l'analyse des lois d'images radar," Traitement du Signal, vol. 19, no. 3, pp. 139-167, 2002.

[7] L. Bombrun and J.-M. Beaulieu, "Fisher Distribution for Texture Modeling of Polarimetric SAR Data," IEEE Geoscience and Remote Sensing Letters, accepted, 2008.

[8] J.-M. Beaulieu and R. Touzi, "Segmentation of Textured Polarimetric SAR Scenes by Likelihood Approximation," IEEE Transactions on Geoscience and Remote Sensing, vol. 42, no. 10, pp. 2063-2072, 0ctober 2004.

[9] J-M. Nicolas, "Application de la transformée de Mellin: étude des lois statistiques de l'imagerie cohérente," in Rapport de recherche, 2006D010, 2006. 